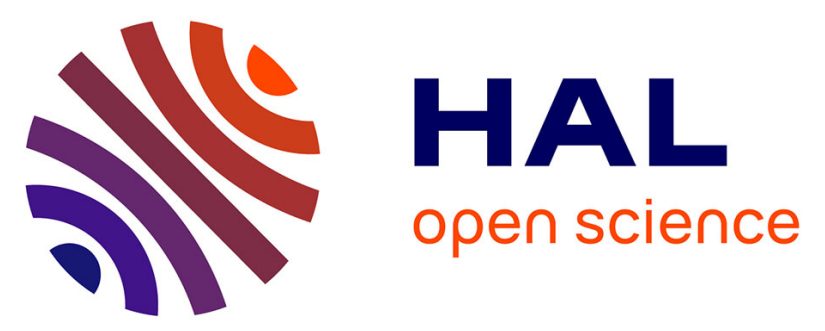

\title{
Studying Gaze Behaviour During Collision Avoidance With a Virtual Walker: Influence of the Virtual Reality Setup
}

Florian Berton, Anne-Hélène Olivier, Julien Bruneau, Ludovic Hoyet, Julien Pettré

\section{To cite this version:}

Florian Berton, Anne-Hélène Olivier, Julien Bruneau, Ludovic Hoyet, Julien Pettré. Studying Gaze Behaviour During Collision Avoidance With a Virtual Walker: Influence of the Virtual Reality Setup. VR 2019 - 26th IEEE Conference on Virtual Reality and 3D User Interfaces, Mar 2019, Osaka, Japan. pp.717-725, 10.1109/VR.2019.8798204 . hal-02058360

\section{HAL Id: hal-02058360 https://hal.inria.fr/hal-02058360}

Submitted on 6 Mar 2019

HAL is a multi-disciplinary open access archive for the deposit and dissemination of scientific research documents, whether they are published or not. The documents may come from teaching and research institutions in France or abroad, or from public or private research centers.
L'archive ouverte pluridisciplinaire HAL, est destinée au dépôt et à la diffusion de documents scientifiques de niveau recherche, publiés ou non, émanant des établissements d'enseignement et de recherche français ou étrangers, des laboratoires publics ou privés. 


\title{
Studying Gaze Behaviour During Collision Avoidance With a Virtual Walker: Influence of the Virtual Reality Setup
}

\author{
Florian Berton* \\ Anne-Hélène Olivier* \\ Julien Bruneau* \\ Ludovic Hoyet* \\ Julien Pettre*
}

Univ Rennes, Inria, CNRS, Irisa, M2S, France
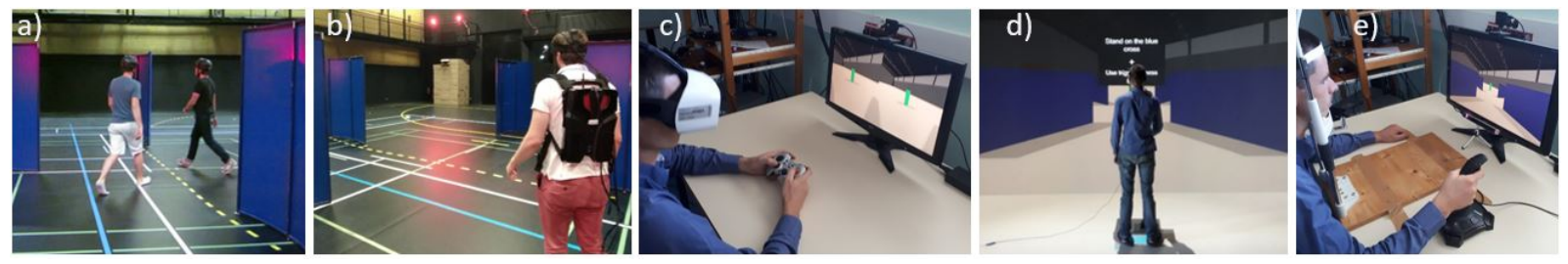

Figure 1: Conditions studied in this paper. (a) Real situation, (b-e) Participants avoiding a virtual character while (b-c) wearing a HMD and (b) walking or (c) navigating using a game controller, (d) standing in a CAVE and navigating using a game controller, (e) interacting with a computer Screen.

\begin{abstract}
Simulating realistic interactions between virtual characters has been of interest to research communities for years, and is particularly important to automatically populate virtual environments. This problem requires to accurately understand and model how humans interact, which can be difficult to assess. In this context, Virtual Reality (VR) is a powerful tool to study human behaviour, especially as it allows assessing conditions which are both ecological and controlled. While VR was shown to allow realistic collision avoidance adaptations, in the frame of the ecological theory of perception and action, interactions between walkers can not solely be characterized through motion adaptations but also through the perception processes involved in such interactions. The objective of this paper is therefore to evaluate how different VR setups influence gaze behaviour during collision avoidance tasks between walkers. To this end, we designed an experiment involving a collision avoidance task between a participant and another walker (real confederate or virtual character). During this task, we compared both the participant's locomotion and gaze behaviour in a real environment and the same situation in different VR setups (including a CAVE, a screen and a Head-Mounted Display). Our results show that even if some quantitative differences exist, gaze behaviour is qualitatively similar between VR and real conditions. Especially, gaze behaviour in VR setups including a HMD is more in line with the real situation than the other setups. Furthermore, the outcome on motion adaptations confirms previous work, where collision avoidance behaviour is qualitatively similar in VR and real conditions. In conclusion, our results show that VR has potential for qualitative analysis of locomotion and gaze behaviour during collision avoidance. This opens perspectives in the design of new experiments to better understand human behaviour, in order to design more realistic virtual humans.
\end{abstract}

Index Terms: Human-centered computing-Visualization-Visualization techniques-Treemaps; Human-centered computingVisualization-Visualization design and evaluation methods

*e-mail: firstname.lastname@inria.fr

\section{INTRODUCTION}

Virtual Reality (VR) is today one important element of the palette of tools to study human behaviour, and more specifically human locomotion, navigation in dynamic environments and interaction with other walkers. Studies have for long focused on kinematics data only, but there is a growing interest to include in addition gaze tracking data because of the fundamental role of the visual perception system in such tasks $[35,42]$. In parallel, affordable consumer VR devices now include gaze tracking possibilities, which raises the question of the usability and validity of VR to perform studies combining motion and gaze tracking data.

The objective of this paper is therefore to evaluate and compare how different VR setups influence gaze behaviour during collision avoidance between walkers. To this end, we designed an experiment involving a collision avoidance task between a participant and another walker, during which we recorded participants' gaze using eye-tracking devices. For comparison, this experiment has been performed in both real and virtual environments using different virtual setups (including a cave, a screen and a Head-Mounted Display).

In the context of collision avoidance task between walkers, the contributions of this article are as follows:

- We propose a methodology based on several objective criteria to evaluate both gaze and kinematic behaviours in virtual and real environments.

- We provide recommendations for the design of VR platforms to perform human locomotion studies in the context of collision avoidance between walkers.

The remaining of the article is organized as follows. Section 2 describes related work with respect to the use of VR and eye-tracking devices to study human interactions during locomotion. Section 3 presents the general description of our experimental study. Section 4 describes the different criteria used to compare the trajectories and gaze behaviour. Finally, results are presented and discussed respectively in Section 5 and 6.

\section{Related Work}

In this section, we first survey previous works that studied interactions between walkers from the kinematics point of view, followed by a focus on gaze behaviours in such tasks. 


\subsection{Kinematics of Interaction Between Walkers}

Human interaction during locomotion and more especially collision avoidance has been extensively studied. Avoidance can be described as the response to a risk of future collision that humans can accurately perceive [33]. Note that this paper describes risk of collision by introducing the $m p d$ function that we use as well in this paper. Avoidance strategies - i.e., how speed and/or orientation adjustments are combined $[3,32]$ - depend more on the characteristics of the situation (e.g. walking speed, angle of crossing) rather than the characteristics of the walkers (e.g., gender, personality) [20,24,32]. Those studies were performed in real conditions (i.e., human participants in a physical environment) and face the difficulty of standardizing situations of interactions, this limited the study of the many factors that can influence such tasks.

VR offers ways to overcome such limitations, e.g., it was used: to study the human behaviour and its neural mechanisms [40], to study locomotor control by manipulating the perceived optical flow $[8,38,43]$, to explore interactions of higher dimension with groups [5] or to evaluate small-scale interaction details such as the effect of mutual gaze and head movements [25,30,41]. Furthermore, nonrealistic scenarios can be studied in VR to isolate and understand specific factors difficult to evaluate in real situations. For instance, Lynch et al. [26] investigated motion cues used during collision avoidance by displaying the avoided agent in different ways, showing the full body motion, global cues only (moving cylinder or sphere) or local cues only (moving legs or trunk only). Finally, VR is also a way to investigate factors while reducing potential risks for participants, typically in specific population such as post-stroke patients $[2,12]$.

The increasing interest in VR tools to perform human experiments stresses the need for validating them, i.e., to compare human behaviour in VR and reality. Several works already performed this comparison for locomotion studies, either considering goal directed $[1,10]$ or collision avoidance tasks $[1,6,14,31]$. It was shown that virtual and real trajectories have common properties, even if some quantitative differences remain such as slower walking speeds or increased clearance distances. In a similar context, Gerin Lajoie et al. [18] found that the personal space during locomotion has a similar elliptic shape but is wider in VR. Moussaid et al. [29] however found that participants seemed to display similar patterns and social conventions. All these works converge to the same conclusion: VR can be used to qualitatively study the kinematics of human locomotion during goal directed trajectories or collision avoidance tasks, while quantitative differences remain.

\subsection{Gaze Behaviour}

Most of previous studies considered trajectories performed by walkers only, few also explored participants' gaze behaviour in relation with trajectory adjustments. Yet, vision plays an important role in the control of locomotion $[35,42]$. In particular, it enables the collection of information about the environment and about the relative movement of surrounding objects.

Using eye-tracking in real environments, it was shown that walkers looked at zones which maximize the level of information that can be used to navigate safely [27]. Cinelli et al. [9] observed participants going through 2 motor-driven sliding doors, and concluded that gaze fixations depended on the complexity of door movements. Few studies considered collision avoidance between walkers. Kitazawa and Fujiyama [23] studied the relationship between gaze and the Personal Space and observed that gaze allocation was equally distributed between ground, objects and pedestrians. Croft et al. [11] studied avoidance strategies between two participants with different velocities, paths and gaze behaviour conditions and found that they predict crossing order. Finally, Jovancevic-Misic and Hayhoe [22] demonstrated that gaze strategies depend on the behaviour of surrounding people, where participants typically looked more at near actors displaying risky behaviours than at other actors.
The integration of eye-tracking capabilities in VR devices such as HMDs greatly facilitate coupled studies of gaze and kinematics data. Jovancevic et al. [21] asked participants to walk in VR among few virtual humans $(\mathrm{VH})$ and they studied the relation between the distribution of gaze fixations in the environment depending on the nature of interactions with $\mathrm{VHs}$, i.e. they focus on following rather than on avoiding. More recently, Meerhoff et al. [28] studied gaze while walking through a crowd in a virtual environment: gaze was more attracted towards the $\mathrm{VH}$ with the highest risk of collision, suggesting a close relation between gaze behaviour and trajectory adaptations. In a different context, VR devices equipped with an eye-tracker have also been used to study human locomotion. For instance, redirected walking techniques [39] recently exploited eye blinks to imperceptibly reorient users. Furthermore, Gandrud et $a l$. [17] found that gaze data activity in relation with head rotation could be used to predict the future direction of a person. This could improve redirected walking techniques, but also improve the realism of avatar's animation.

As for locomotor trajectories, it is necessary to investigate the similarities and biases induced by VR on gaze behaviour if we aim at using VR to draw conclusions on human behaviour. Foulsham et al. [15] studied participants' gaze behavior while walking in a university campus or watching first person videos of this walk. Differences were noticed regarding eye and head movements, however scene objects were mostly looked at in the same proportions. Similar gaze behaviors were also found during experiments conducted in both virtual and real environments where participants sat on a chair and observed either a realistic avatar [37] or a light [36], despite differences in head rotations [36]. To the best of our knowledge, no study compared gaze behaviour during a collision avoidance locomotor task in real and virtual environments. This is however a daily situation of interest to understand how humans interact together. The purpose of our study is therefore to evaluate and compare gaze behaviour during a collision avoidance task between walkers in real and virtual conditions.

\section{Methods}

We designed an experiment to evaluate and compare gaze behaviour during a collision avoidance task. We considered a real baseline condition as well as four virtual conditions. Virtual conditions varied by the type of visual display (Cave, HMD or screen). As it has been demonstrated that participants' gaze can be impacted by action requirements [13], we also considered two types of navigation techniques (physically walking or using a game controller). Our hypotheses are:

- H1: the nature of the visual information retrieved from the environment to achieve the collision avoidance task (i.e., gaze allocation) will be similar between the real and the virtual conditions, similarly to what was previously observed for locomotion behaviour between VR and real conditions. Moreover, we hypothesize that gaze allocation will not be affected by the type of VR display.

- H2: the type of display will however affect gaze movements. Since each type of display does not convey visual information in the same way (e.g., HMD and screen have limited field of view), we expect that gaze movements will adapt accordingly. Especially, we expect that displays with limited field of view will induce larger head (HMD) or eye movements (Screen because we used a chin rest) to explore the environment and compensate for the limited field of view.

- H3: the type of navigation controller will not affect gaze behaviour. Again based on previous studies that demonstrate that locomotion is performed similarly in real and virtual conditions in spite of the use of various locomotion techniques, we 
expect that the nature of the visual information to control virtual locomotion will remain similar, and therefore will induce similar gaze behaviour compared to the real baseline condition.

\subsection{Participants}

Seventeen unpaid participants, recruited via internal mailing lists among students and staff, volunteered for the experiment $(6 \mathrm{~F}, 11 \mathrm{M}$; age: avg. $=23.6 \pm 3.3, \min =19, \max =29$ ). They were all naive to the purpose of the experiment, had normal or correct-to-normal vision, and gave written and informed consent. The study conformed to the declaration of Helsinki, and was approved by the local ethical committee. Because of tracking issues that appeared specifically for five participants (motion capture tracking issues, or eye gaze calibration issues), only the data from twelve participants was finally used in this paper.

\subsection{Task}

Participants were asked to navigate in a real or virtual environment towards a target while avoiding any collision with another walker crossing their way (a virtual character or a real confederate). As shown in Figure 2, the other walker had an orthogonal trajectory to the one of the participant, he could come either from the right- or lefthand side, he walked straight at constant speed (with no adjustment of his trajectory). Participant reached a target in front of them, visible from the beginning. Walls hid the walker to participants at the beginning of the trial to let them reach their comfort speed before they react to the virtual walker. We varied the risk of collision with the other walker by defining 13 offset positions for the walker, that resulted into advance or delay on the participants' motion (6 giving advance, 6 delaying him and 1 symmetrical situation with full risk of collision).

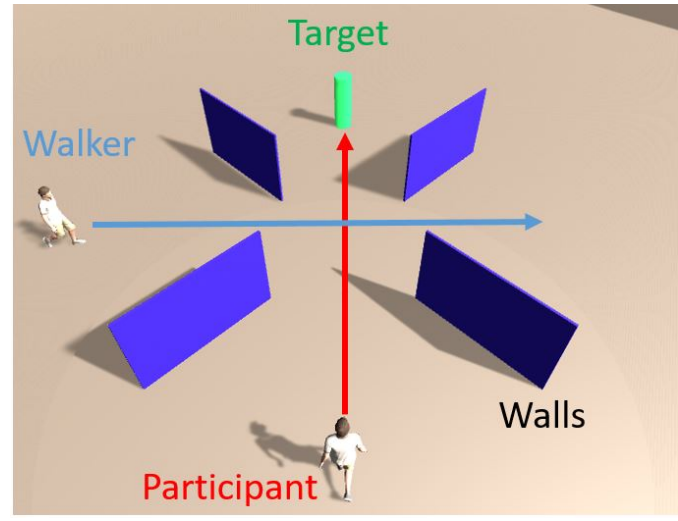

Figure 2: Participants were asked to navigate towards a target, while avoiding any collision with a real or virtual confederate crossing their path perpendicularly. Walls prevented participants from seeing the real or virtual confederate before reaching their comfort speed.

\subsection{Environment}

Participants performed the task in a real and a virtual environment.The real environment was delimited by a $9 m x 9 m$ square, with four walls placed along its the diagonal at $2.2 \mathrm{~m}$ from the center (see Figure 2). They have to reach the opposite corner of the square. Furthermore, the confederate walker did not perform eye contact and did not react to participants.

In comparison, virtual conditions took part in a virtual copy of the physical environment (dimensions, start and target positions, walls) which we designed in Unity 2017.4. The target represented as a green cylinder. The virtual walker based animated based on motion captures of the real confederate. Note that animations did not include gaze movement or facial expression to keep similar behaviour of the walker with respect to the real condition.

\subsection{Experimental Design}

Participants were asked to perform the task presented in Section 3.2 in the five following conditions (summarized in Table 1):

Real: this is our baseline condition to compare VR situations to. Participants were asked to walk at comfort speed and to avoid any collision with the confederate walker. A 28-camera Vicon system $(120 \mathrm{~Hz})$ was used to record their trajectory, where participant and confederate positions were approximated as the centre of their head using bike helmets equipped with reflective markers. Participants also wore Tobii eye-tracking glasses which recorded both their gaze behaviour $(50 \mathrm{~Hz})$ and what they saw of the environment (scene camera: $25 \mathrm{~Hz}, 90^{\circ}$ field of view).

HMD-W: participants were immersed in the virtual environment using a FOVE HMD $\left(70 \mathrm{~Hz}, 100^{\circ}\right.$ field of view), which comes with an integrated eye-tracker ${ }^{1}(100 \mathrm{~Hz})$. They performed the collision avoidance task with the virtual character by walking in the co-localised physical setup (Figure 1.b). As for the Real condition, the 28-camera vicon system was used to track participants' trajectories using markers positioned on the HMD, as well as to update the participants' viewpoint in the virtual environment.

HMD-C: participants were immersed in the virtual environment using a FOVE HMD (see above), which was also used to record their gaze behaviour. However, in this condition we were interested in a different navigation technique, where participants used a game controller (Logitech F710 S) to avoid collisions with the virtual character, while seated at a desk. With no action on the controller, participants moved straight with a default speed of $1.33 \mathrm{~m} . \mathrm{s}^{-1}$ (comfort walking speed from [4]). They were able to linearly adapt their speed from $0.8 m \cdot s^{-1}$ to $2 m \cdot s^{-1}$ with the longitudinal axis and their angular speed from $-25 \mathrm{deg} . \mathrm{s}^{-1}$ to $25 \mathrm{deg} . \mathrm{s}^{-1}$ with the lateral axis of the controller. This control scheme was previously validated by Olivier et al. [31] for such collision avoidance tasks.

Cave: participants were immersed in the virtual environment using a 4-screen Computer Assisted Virtual Environment (CAVE). the system is equipped with 13 projectors, has a 15MPixels resolution in total, and is $9 \mathrm{~m}$ large, $3 \mathrm{~m}$ high and $3 \mathrm{~m}$ deep. Participants wore volfoni 3D glasses for active stereo vision, as well as the eye-tracking Tobii glasses, assembled with a custom-built rig. Participants were standing in the middle of the CAVE and asked to avoid collisions with the virtual character using a joystick (Logitech Attack 3), which used the same control scheme described above.

Screen: participants sat at a desk in front of a 24-inch screen, and were asked to avoid collisions with the virtual character using a joystick (Logitech Attack 3), which used the same control scheme described above. Gaze behaviour was recorded using The Eyetribe $(60 \mathrm{~Hz})$ positioned under the screen, and a chin rest was used to increase tracking accuracy.

Participants performed the experimental task for each of these conditions. This was combined with 13 levels of initial risk of collision with the confederate or the virtual walker. Finally, we included two repetitions of each situation, where the walker to avoid can come from the left or from the right. In total, each participant performed 130 trials $(5$ Conditions $\times 13$ Risk levels $\times 2$ Repetitions).

${ }^{1}$ It is important to note that we used three different models of eye-trackers in this experiment (Tobii glasses, Eyetribe desktop eye-tracker, and FOVE built-in eye-tracker) to fit the constraints of the VR devices. E.g., it was not possible to use eye-tracking glasses in the HMD, and we therefore used the built-in device. 


\begin{tabular}{l|c|c} 
Condition & Eye-tracker & Navigation \\
\hline \hline Real & Tobii & walking \\
HMD-W & Fove (built-in) & walking \\
HMD-C & Fove (built-in) & game controller \\
CAVE & Tobii & game controller \\
Screen & Eyetribe & game controller
\end{tabular}

Table 1: Summary of the conditions presented in this experiment

They were also invited to perform up to 20 additional training trials at the beginning of each condition (most participants performed 4 to 5 in each case).

\section{Analysis}

\subsection{Collected Data}

During the experiment, the participant and the walker trajectories were recorded, as well as participants' head rotation and gaze behaviour (origin and direction). The image of what the participant saw (Figure $3 \mathrm{a}-\mathrm{b}$ ) was recorded either with the Tobii glasses for the real environment, or with Unity for the virtual environment. This image was divided into three different items that could be targeted by the gaze (Walker, Target or Environment). This segmentation (Figure $3 \mathrm{c}-\mathrm{d}$ ) was done either using shaders in Unity for the virtual environment, or manually with the help of a CNN network [7] on the gaze video for the real environment. Furthermore for each trial, we re-sampled the recorded data (trajectories, eye movements) at a frequency of $60 \mathrm{~Hz}$.
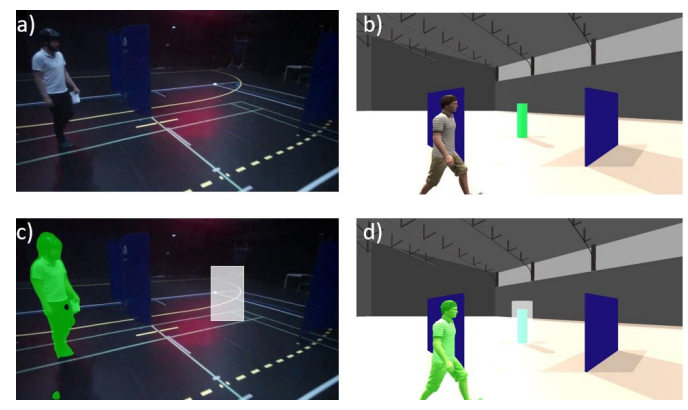

Figure 3: Real and virtual environments (a-b) and their respective segmentation (c-d). Walker is in green, Target in white and the rest is considered as Environment

\subsection{Time Variables}

To describe the interaction period ${ }^{2}$, we defined two variables: the moment when the confederate walker is not obstructed by the wall anymore, which corresponds to the first instant when the participant can first see him (Tsee), and the moment when the two walkers are the closest to each other (Tcross). We then normalized the time along this interaction period from $0 \%$ (T see) to $100 \%$ (Tcross).

\subsection{Collision Avoidance Trials}

The goal of this paper is to study gaze behaviour during a collision avoidance task. We therefore needed to distinguish trials where a collision avoidance was performed (i.e., the initial risk of collision was high enough to trigger collision avoidance manoeuvres) from those that did not require trajectory adjustments. To this end, we based our analysis on the minimum predicted distance ( $m p d$ ) [33],

${ }^{2}$ The term 'interaction' has been used in previous work to provide a temporal description of the task. Even though the virtual character is not reactive, and thus, the interaction is not really present in our experiment, we decided to keep calling this period 'interaction' for the sake of clarity which can be computed at any time $t$ between Tsee and Tcross. At any time $t, \operatorname{mpd}(t)$ therefore corresponds to the closest distance the two walkers would be passing by in the future if they were to maintain their current velocity. Any variation of $m p d$ in time necessarily results from motion adaptions performed by walkers.

More specifically, these two types of trials (with or without avoidance manoeuvres) will differ in the evolution of $m p d(t)$. Trials with trajectory adjustments to avoid collision typically have a significant increase of $m p d$ in time, i.e., $m p d($ T see $)<m p d$ (Tcross), while the latter type of trials typically has a more constant $m p d$, with the exception of the effect of trajectory noise (e.g., swaying in locomotion), i.e., $m p d($ Tsee $) \approx m p d($ Tcross $)$. As a result, a threshold $m p d_{C A}$ on the value of $m p d$ (T see) over which Collision Avoidance is not necessary anymore can be identified in order to divide our data into trials with collision avoidance and trials without collision avoidance. This threshold was automatically computed by fitting the following model describing the evolution of $m p d$ (Tcross) in relation to $m d p$ (Tsee) on our data (see Figure 4 for illustrative representation):

$$
\begin{gathered}
\operatorname{minimize}_{a, m p d_{C A}} \sum_{i}^{N}\left(f\left(\text { Tsee }^{i}\right)-\text { Tcross }^{i}\right)^{2} \\
\text { subject to } f(x)=\left\{\begin{array}{cl}
a \times x+b & \text { if } x<m p d_{C A} \\
x & \text { subj. to } b=(1-a) \times m p d_{C A}
\end{array}\right.
\end{gathered}
$$

It is important to note that we computed the best parameters $a$ and $m p d_{C A}$ which minimized the sum of squared residuals (SSR) independently for each condition, in order to compute a threshold adapted to the data from each condition.

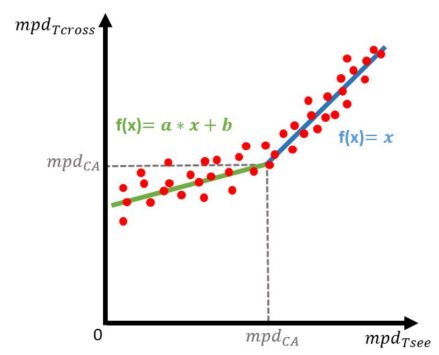

Figure 4: Schematic model used to calculate $m p d_{C A}$

\subsection{Kinematics of the collision avoidance task}

In the real condition, we asked the confederate not to react to participants. We assessed the absence of reaction by computing his acceleration during the interaction period.

Then, for all conditions, we analyze the kinematic characteristics of the collision avoidance task by computing for each trial:

- Number of collisions: we defined a collision as occurring when the distance between the two walkers (computed from center to center) is less than $50 \mathrm{~cm}$.

- Number of inversions:by linearly extrapolating the participant trajectory from current positions and velocities, we can estimate the future crossing order at time Tcross. We count the number of trials for each condition where an inversion of this order occurs along the trial.

For trials were there was a collision avoidance, we computed:

- Clearance Distance: the actual distance (in $\mathrm{m}$ ) between the two walkers at $T$ cross (i.e., $\left.\operatorname{md} p\left(T_{\text {cross }}\right)\right)$ ).

- Speed : average participant's speed over the interaction period.

- Mpd evolution: to characterize trajectories adaptations, we computed the $m p d$ evolution during the normalized interaction as well as its temporal derivative. 


\subsection{Gaze Behaviour}

As our goal in this paper is to understand gaze behaviour leading to avoidance adaptations, we analyzed participants' gaze behaviour only for trials with motion adaptations. We considered two main aspects of participants' gaze behaviour namely fixations, and head and eyes movements. In the case of fixations, we also define the gaze allocation as the ratio of time spent looking at each object during the whole interaction.

Fixation. According to Parasuraman and Rizzo [34], the gaze behaviour can be described as a succession of fixations separated by fast eye movement called saccades. An important task in eyetracking studies is to well define these two movements [19]. Depending on the task and situation different definitions can be found in the literature, and we therefore used the definition of fixations given by Kitazawa and Fujiyama [23], whose experimental task shared common properties with ours: a fixation was defined as a continuous gaze on the same object for more than $80 \mathrm{~ms}$. Furthermore, for each temporal window of $80 \mathrm{~ms}$, all the gaze points were required to be within a range of 3.0 degrees from the initial point. Considering this definition, our dependent variables related to fixations during the interaction were:

- Number of fixations per second

- Average duration of fixations in seconds.

- Gaze Allocation: we reported, in \%, where participants looked at considering 3 allocations: the confederate walker, the target and the surrounding environment.

Gaze and head angles. To consider gaze and head movements during the interaction period, we computed the following angles in the $X Y$ plan, where both the confederate walker and the target were represented as a circle with respectively a radius of $25 \mathrm{~cm}$ and $50 \mathrm{~cm}$ :

- Angle ${ }_{H H}$ : angle between the head vector and the heading. It was not computed for the Screen condition as participants head was immobilized using a chin rest, and therefore always aligned with the heading direction.

- Angle ${ }_{G H}$ : angle between the gaze vector and the head vector.

- Angle ${ }_{G W}$ : angle between the gaze vector and the other walker.

- Angle $_{G T}$ : angle between the gaze vector and the target.

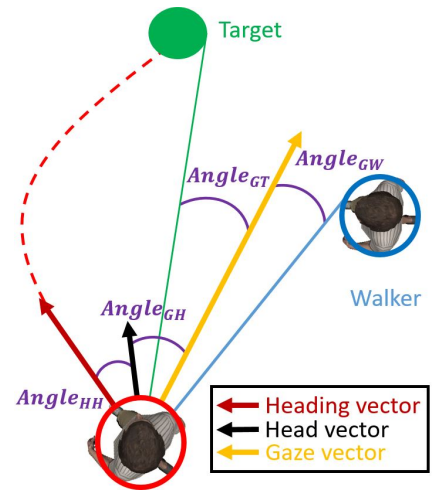

Figure 5: Illustration of the 4 angles we computed to relate head and eye movements.

\subsection{Statistics}

For all dependent variables, we set the level of significance to $\alpha=0.05$. A Shapiro Wilk test was performed to evaluate whether data followed a normal distribution. If the distribution was not normal, a Friedman test was performed to evaluate the effect of the condition on these variables. Post-hoc comparisons were performed using a Wilcoxon signed rank test with a Bonferroni correction. If the distribution was normal, a one-way analysis of variance (ANOVA) with repeated measures was performed. GreenhouseGeisser adjustments to the degrees of freedom were applied, when appropriate, to avoid any violation of the sphericity assumption. Bonferroni post-hoc tests were used to further analyze significant effects. For all variables which described an evolution during the normalized time of interaction, we evaluated the effect of the condition using Statistical Parametric Mapping (SPM) methods [16]. This analysis allows comparing time-series data of different trials taking into account their variability at each time-step.

\section{Results}

\subsection{Locomotion}

In the real condition, we confirmed that the confederate walker had approximately a constant speed ( $1.4 \pm 0.1 \mathrm{~m} \cdot \mathrm{s}^{-1}$ in average).

Thresholds for adaptation We computed the threshold $m p d_{C A}$ separately for each condition, and trials where $\operatorname{mpd}\left(T_{s e e}\right)$ was lower than $m p d_{C A}$ of the condition. Trials were then considered to contain trajectory adjustments to avoid a potential collision. The threshold for each condition are reported in Table 2. Overall, we found a lower threshold for the real condition than for the virtual conditions, which is further discussed in Section 6.

Average speed of participants (Cf.,Table 2) was affected by the condition $\left(F(1.72,18.88)=10.12, p<0.0001, \eta^{2}=0.48\right)$, with lower values observed for the HMD-W condition.

Table 2: $m p d_{C A}$, average $( \pm S D)$ speed of participants, average $( \pm S D)$ number of collisions, and average $( \pm S D)$ number of inversions of crossing order with respect to the experimental conditions.

\begin{tabular}{|l|c|c|c|c|}
\hline Conditions & $\begin{array}{c}m p d_{C A} \\
(\mathrm{~m})\end{array}$ & $\begin{array}{c}\text { Speed } \\
\left(\mathrm{m} \cdot \mathrm{s}^{-1}\right)\end{array}$ & Collisions & Inversions \\
\hline Real & 0.97 & $1.34 \pm 0.16$ & $0.16 \pm 0.38$ & $1.00 \pm 0.85$ \\
\hline HMD-W & 1.40 & $1.08 \pm 0.16$ & $0.17 \pm 0.39$ & $1.58 \pm 1.08$ \\
\hline HMD-C & 1.29 & $1.27 \pm 0.07$ & $0.25 \pm 0.45$ & $0.50 \pm 0.67$ \\
\hline CAVE & 1.40 & $1.25 \pm 0.05$ & $0.33 \pm 0.65$ & $1.16 \pm 0.83$ \\
\hline Screen & 1.46 & $1.23 \pm 0.06$ & $0.58 \pm 0.90$ & $1.00 \pm 0.85$ \\
\hline
\end{tabular}

Number of collisions and inversion are illustrated in Table 2. These values were low (NB, values computed for each participant on the 26 trials of each condition). Furthermore, there is no significant effect of the condition on the number of collisions $(\mathrm{p}=0.79)$ or inversions $(p=0.26)$.

Clearance Distance, presented in Figure 6, was affected by the condition $\left(F(2.35,25.92)=16.72, p<0.0001, \eta^{2}=0.60\right)$. Bonferroni post-hoc tests showed that it was lower in the real condition than in all the conditions performed in a virtual environment.

Mpd evolution and its time derivative during the interaction period are illustrated in Figure 7. Qualitatively, we can notice that all the curves have similar shapes. From a quantitative point of view, SPM analysis showed an effect of the condition on mpd values during all the interaction $(p<0.001)$. Post hoc tests revealed that mpd in real conditions was lower than HMD-C, Screen and Cave conditions during all the interaction period, and than HMD-W from $25 \%$ to the end of the interaction $(p<0.001)$. No effect of the condition was however shown when considering mpd time derivative. 


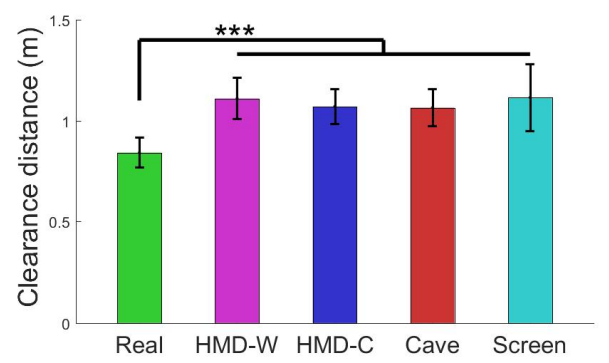

Figure 6: Average clearance distance $( \pm S D)$ depending on the experimental condition. Significant post-hoc comparisons are highlighted with stars $\left({ }^{* * *} \mathrm{p}<0.001\right)$
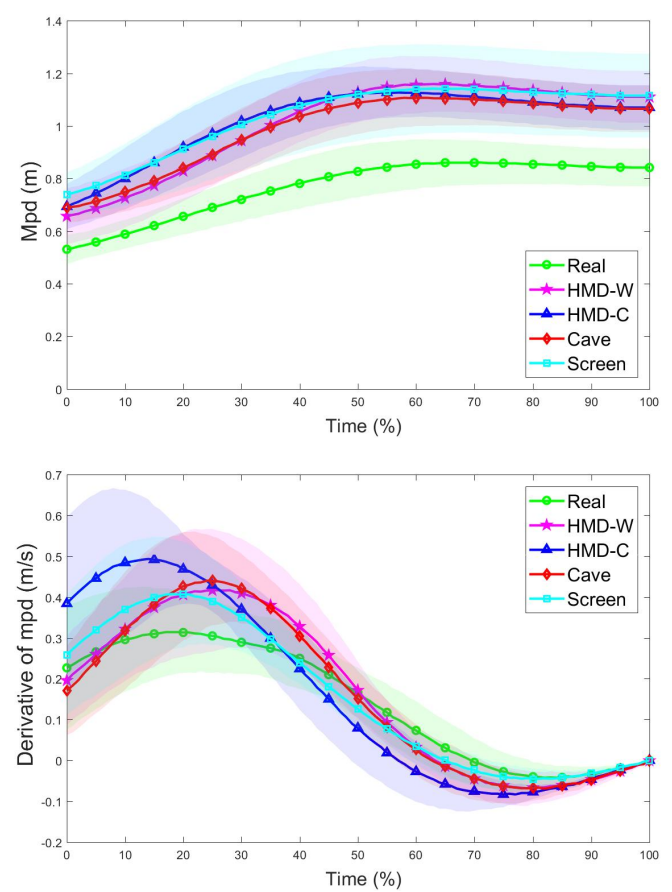

Figure 7: Kinematics adaptations to avoid a collision during the interaction are depicted through minimum predicted distance (mpd) (top) and its temporal derivative (bottom).

\subsection{Gaze Behaviour}

\subsubsection{Fixations}

Number of fixations per second during the interaction period is illustrated in Figure 8. A significant effect of the condition was observed $\left(F(4,44)=4.88, p=0.0024, \eta^{2}=0.31\right)$. Post-hoc analysis showed that there were less fixations in the Cave than in the conditions involving physical walking, namely HMD-W and Real.

Average duration of fixation is illustrated in Figure 9 and was influenced by the condition $\left(\chi^{2}(4)=28.46, p<0.0001\right)$. It was longer for the Screen than for the Cave and the HMD-W, smaller for the Cave than the HMD-C and smaller for HMD-W than HMD-C.

Gaze allocation is summarized in Figure 10. A significant effect of the condition was observed on the ratio of fixation directed towards the confederate walker $\left(F(4,44)=4.25, p=0.0053, \eta^{2}=0.28\right)$, the target $\left(F(4,44)=3.67, p=0.011, \eta^{2}=0.25\right)$, as well as the en-

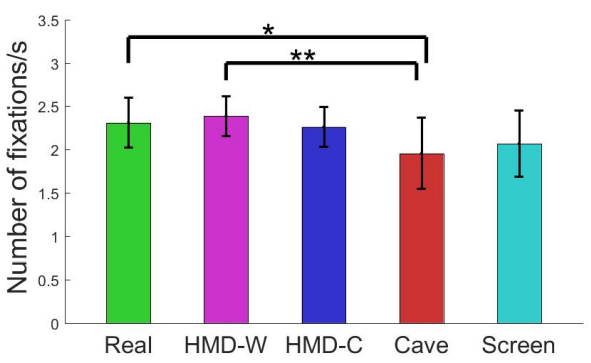

Figure 8: Number of fixations per second during the interaction for each condition

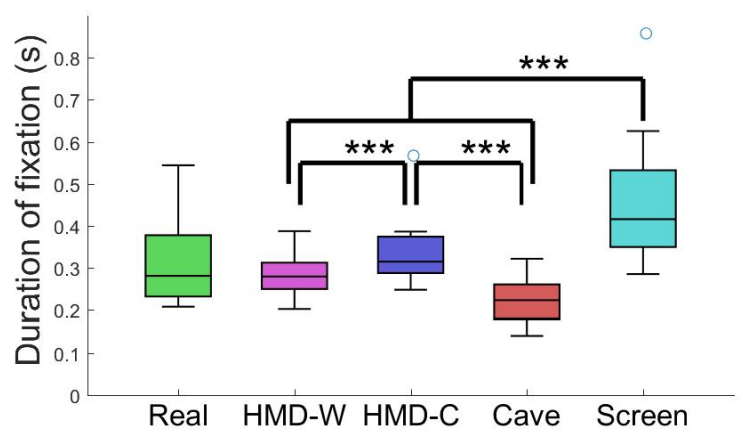

Figure 9: Average duration of fixation during the interaction for each condition.

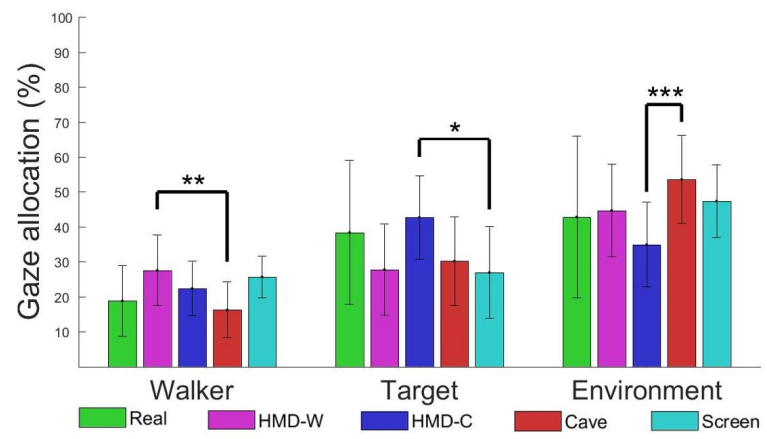

Figure 10: Average $( \pm S D)$ gaze allocation towards the confederate walker (left), the target (center) and the environment (right) during the interaction period.

vironment $\left(F(4,44)=3.45, p=0.015, \eta^{2}=0.24\right)$. Post-hoc analysis showed that gaze allocation towards the confederate walker was lower in the Cave than in the HMD-W condition. Gaze allocation towards the target was higher in the HMD-C than in the Screen condition. Finally, gaze allocation towards the environment was lower in the HMD-C than in Cave condition.

\subsubsection{Gaze and head angles}

Figure 11 illustrates the evolution during the interaction period of all the angles related to gaze and head. SPM analysis showed an effect of the condition for all angles. Significant differences from the post-hoc SPM analysis are depicted on the top of each sub-figure by an horizontal bar on the corresponding period of the interaction.

Angle $_{H H}$ in Real conditions was significantly smaller than for HMD-W during the first half of the interaction, and than for the 

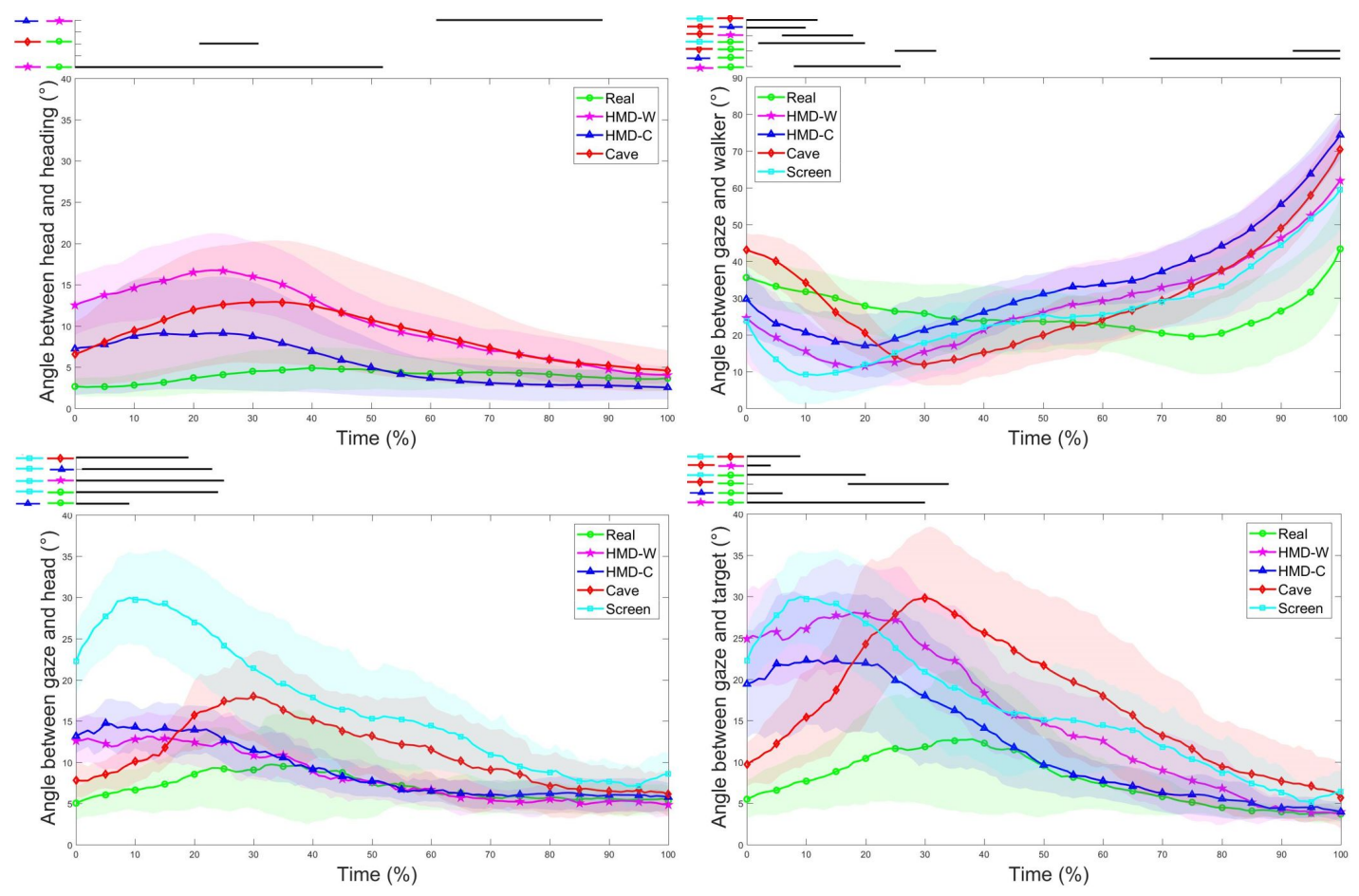

Figure 11: Evolution of the four angles Angle ${ }_{H H}$ (top left), Angle ${ }_{G H}$ (bottom left), Angle ${ }_{G W}$ (top right) and Angle ${ }_{G T}$ (bottom right) during the normalized interactions for all the conditions. SPM analysis showed an effect of the condition on each angle. Significant pairwise post hoc results are depicted above each figure where horizontal black lines indicate during which period of time the angle differs between 2 conditions.

Cave from $21 \%$ to $31 \%$ of the interaction period. Also, angle ${ }_{H H}$ was bigger in HMD-W than in HMD-C at the end of the interaction.

Angle $_{G H}$ in Screen conditions was significantly bigger (up to twice) than in all the other conditions at the beginning of the interaction. A significant difference was also noticed between the Real and the HMD-C conditions, angle ${ }_{G H}$ being bigger in the HMD-C conditions from $0 \%$ to $9 \%$ of the interaction.

Angle $_{G W}$ in Real conditions at the beginning of the interaction was bigger than in HMD-W and Screen conditions and smaller than in the Cave conditions. It was also smaller than in the HMD-C and the Cave conditions at the end of the interaction. Also the angle ${ }_{G W}$ in the Cave conditions was bigger than all the other three VR conditions at the beginning of the interaction.

Angle $_{G T}$ in Real conditions at the beginning of the interaction was smaller than all the VR conditions. It was also smaller at the beginning of the interaction in Cave compared to Screen and HMD-W.

\section{Discussion}

The main objective of this study was to evaluate and compare the influence of various VR setups on the gaze behaviour during a collision avoidance task. To this end, we designed an experiment were participants performed the task in real and virtual conditions and we tracked both their trajectory and gaze.

\subsection{Collision avoidance behaviour}

Kinematics analysis showed that there were some quantitative differences in the metrics of interaction between real and virtual environments. Especially, the threshold $m p d_{C A}$ that triggers motion adaptation was lower in real condition than in virtual conditions, which is consistent with the larger clearance distance observed in the VR conditions. However, when considering the derivative of $m p d$, i.e., $m p d$ variations and not absolute values, the absence of significant difference between the studied conditions suggests that motion adjustments are performed similarly in both real and virtual environments. This corroborates results from previous studies [6, 14, 31]. Furthermore, considering the VR conditions only, we did not find any significant difference between $m p d$ values along the whole collision avoidance task. This suggests that collision avoidance behaviour is similar regardless of the employed VR setup. We found a difference in the average walking speed that was lower in the HMD-W condition: participants wore a HMD but had to move among physical obstacles, which may have induced a safer locomotion speed. This result is also consistent with previous works $[1,6]$.

\subsection{Fixations and gaze allocation}

Apart from the number of fixations in the Cave condition, our results showed similarities in the number and duration of fixations between real and virtual conditions. The smaller number of fixations in the Cave condition may be linked to technical issues where we found that eye gaze recognition was slightly impaired by the lighting conditions (which is specifically discussed in Section 6.4).

Regarding gaze allocation (i.e., ratio of what participants looked at in the scene), results only showed a few statistical differences between the VR conditions (Figure 10), where participants looked longer on average at the walker in one condition than another (HMD-W > Cave), or looked longer on average at the target (HMD-C $>$ Screen). However we did not find any significant difference between the Real condition and any of the VR conditions.

Overall, our results validate our Hypothesis H1. Comparing Real and VR conditions, participants looked at a similar visual content. They spend similar proportion of time on the various elements of the scene, with similar visual patterns. However we observed a few differences between the studied VR setups. This indicates that in spite of significant difference with Real conditions, some setups 
tend to change gaze behaviours in Virtual Reality and could become more important when studying other type of tasks for example. We discuss more extensively those limitations below.

\subsection{Gaze and head angles}

While the evaluation of what participants look at during the interaction is important, another interesting aspect is to evaluate whether the way they looked at this content was similar in the studied conditions.

First, we measured the evolution of the angle between the participants' gaze vector and both the walker and target, respectively by computing Angle $_{G W}$ and Angle e $_{G T}$. We observed that most of the differences between the angle evolution appeared at the start of the interaction, where participants could first see the other walker (namely, the observation phase [33]). We can observe in Figure 11 (top and bottom right) an overall decrease of Angle $_{G W}$ with a simultaneous increase of Angle $_{G T}$, showing that participants tended to look progressively more towards the walker and less towards the target in the first part of the interaction. As the walker is perceived with a possible risk of collision, this pattern is in correlation with previous work [22,28]. However, we observed that the amplitude of these two variations was smaller in the real condition compared to the VR conditions, suggesting that peripheral vision might have played an important role in the real condition. These two variations also seem to happen later for the Cave and Real conditions than for the other VR conditions, which again seem to demonstrate the effect of the larger peripheral vision available in these two conditions. Therefore, it is likely that the limited peripheral vision in the HMD conditions forced participants to actively look for the other walker earlier in the interaction than in the Cave and Real conditions.

Then, we evaluated how participants' gaze direction resulted from the Eye-Head-Body relative orientations. In particular, we explored how the angle of the head relative to the heading evolved over time $\left(\right.$ Angle $_{H H}$ ), as well as the angle of the gaze relative to the head $\left(\right.$ Angle $\left._{G H}\right)$. We found statistical differences at the beginning of the interaction between the Real condition and any of the VR conditions, for either Angle ${ }_{H H}$ or Angle ${ }_{G H}$, which is in correlation with previous work [36] for a stationary situation. In the case of the HMD-W and Cave conditions, statistical differences were found only for Angle $_{H H}$, differences which were longer in time for HMD-W. Conversely, for the Screen and HMD-C conditions statistical differences were found only for Angle $_{G H}$. This seems to show that participants moved more their head in relation to their body in the HMD-W and Cave conditions, but displayed similar Eye-Head patterns. These results validate H2. Because of the strong differences for HMD-W we hypothesize that such differences in behaviour might have been caused by the limited field of view of the HMD, which might have forced participants to induce larger head motions to look for the walker at the beginning of the interaction. This hypothesis should be evaluated through further studies. Similarly, such differences in the Screen condition were expected as participants' head was restricted because of the use a chin rest (required to improve the accuracy of the eye-tracker), which would have caused them to perform the scene exploration only using their eyes.

The results on Angle $_{G H}$ between the HMD-C and Real conditions were however more surprising, especially as we expected the limited field of view to lead to similar patterns than for HMD-W. This difference on Eye-Head-Body relative orientations between HMD-W and HMD-C when compared to the Real condition could therefore also be due to the difference in the locomotion interface, namely walking or seating using a game controller. This suggests that not only the type of VR device, but also the locomotion interface, might have an impact on the Eye-Head-Body coordination, which partially invalidates $\mathbf{H 3}$ (at least in terms of differences in eye movements). Therefore, further studies should be conducted to thoroughly evaluate these effects.

\subsection{Limitations}

Even with high-end eye-tracking devices, accurately tracking eye gaze can be influenced by the experimental conditions, such as lighting or participant's eye color. For the experiment reported in this paper, data from four participants were discarded because of eye-tracking issues regarding the quality of the calibration (3 participants with the Tobbi glasses, 1 participant with the FOVE HMD). In particular, we found the Tobbi glasses to be very sensitive to the room illumination while tracking the gaze, both in the Real and Cave situations. While this impacted calibration for three discarded participants, we also found that it led to more noise in the data in the Cave condition across participants, including the inability to identify eye gaze in some frames and influencing the identification of fixations in the data. We believe that such a problem might explain at least partially the lower average of fixations per second for the Cave condition (Figure 8), where illumination is typically reduced for $3 \mathrm{D}$ projection purposes. Regarding gaze tracking issues for the one participant in the HMD-W condition, we hypothesize that it could come from the head movements, a combination of head movements and a HMD not tightly fixated, or bad calibration because of eye color. These technical limitations therefore influenced our sample size, which should be increased in future experiments to explore more subtle effects and to strengthen our conclusions.

While the presented experiment is to our knowledge the first attempt to study in VR the kinematics of a collision avoidance task in conjunction to gaze behaviour, it also involves a specific controlled interaction in restricted physical and virtual spaces. For instance, interactions were possible in a $9 \mathrm{~m} x 9 \mathrm{~m}$ area, which can also influence participant behaviour. Therefore, further studies would benefit from evaluating kinematics and gaze behaviours in larger physical setups, as well as more complex situations.

\section{CONCLUSION \& FUtURE WORK}

In this paper, we explored the use of VR to study locomotion and gaze behaviour during collision avoidance between walkers, where we studied a collision avoidance task between two walkers in both real and virtual environments. We then compared the real condition with four VR conditions (including a CAVE, a screen and a Head-Mounted Display). Our results show that fixations and gaze allocations were similar in both real and virtual environments. However the exploration of the environment, to seek visual information, was different in both real and virtual environments. Our results suggest that VR has potential to study qualitatively the gaze behaviour, even though it does not seem to be befitting to study accurately the movement of the eyes with the current technological setups. Furthermore, not only the type of VR device but also the locomotion interface has an impact on eye movement, which should therefore also be taken into consideration. Regarding motion adaptations, our outcome confirms previous work, where collision avoidance behaviour is qualitatively similar in VR and real conditions.

In the future we want to look deeper into these effects, such as evaluating the impact on the crossing order, or the relationship between gaze behaviour and body motions. As VR also offers the possibility to explore complex or even inextricable situations while preserving qualitative human behaviours, we also want to explore further such complex scenarios in the future, e.g., by considering situations such as walking in a crowd, such as [28], while manipulating different factors. In line with previous study, participants' gaze activity provides precious insights about individual behaviours in crowds, the nature of information acquired from the environment to achieve crowd behaviours, and the understanding of complex interactions in such contexts.

\section{ACKNOWLEDGMENTS}

This work was funded by the ANR OPMoPS project (ANR-16SEBM-0004). 


\section{REFERENCES}

[1] P. Agethen, V. S. Sekar, F. Gaisbauer, T. Pfeiffer, M. Otto, and E. Rukzio. Behavior analysis of human locomotion in the real world and virtual reality for the manufacturing industry. ACM Transactions on Applied Perception (TAP), 15(3):20, 2018.

[2] G. Aravind, A. Darekar, J. Fung, and A. Lamontagne. Virtual realitybased navigation task to reveal obstacle avoidance performance in individuals with visuospatial neglect. IEEE Transactions on Neural Systems and Rehabilitation Engineering, 23(2):179-188, 2015.

[3] P. Basili, M. Sağlam, T. Kruse, M. Huber, A. Kirsch, and S. Glasauer Strategies of locomotor collision avoidance. Gait \& posture, 37(3):385390, 2013.

[4] R. W. Bohannon. Comfortable and maximum walking speed of adults aged 20-79 years: reference values and determinants. Age and ageing, 26(1):15-19, 1997.

[5] J. Bruneau, A.-H. Olivier, and J. Pettré. Going through, going around: A study on individual avoidance of groups. IEEE transactions on visualization and computer graphics, 21(4):520-528, 2015.

[6] M. A. Bühler and A. Lamontagne. Circumvention of pedestrians while walking in virtual and physical environments. IEEE Transactions on Neural Systems and Rehabilitation Engineering, 2018.

[7] S. Caelles, K.-K. Maninis, J. Pont-Tuset, L. Leal-Taixé, D. Cremers, and L. Van Gool. One-shot video object segmentation. In Computer Vision and Pattern Recognition (CVPR), 2017.

[8] Y.-h. Chou, R. C. Wagenaar, E. Saltzman, J. E. Giphart, D. Young, R. Davidsdottir, and A. Cronin-Golomb. Effects of optic flow speed and lateral flow asymmetry on locomotion in younger and older adults: a virtual reality study. Journals of Gerontology: Series B, 64(2):222-231, 2009.

[9] M. E. Cinelli, A. E. Patla, and F. Allard. Behaviour and gaze analyses during a goal-directed locomotor task. The Quarterly Journal of Experimental Psychology, 62(3):483-499, 2009.

[10] G. Cirio, A.-H. Olivier, M. Marchal, and J. Pettré. Kinematic evaluation of virtual walking trajectories. IEEE transactions on visualization and computer graphics, 19(4):671-680, 2013.

[11] J. L. Croft and D. Panchuk. Watch where you're going? interferer velocity and visual behavior predicts avoidance strategy during pedestrian encounters. Journal of Motor Behavior, 0(0):1-11, 2017.

[12] A. Darekar, A. Lamontagne, and J. Fung. Dynamic clearance measure to evaluate locomotor and perceptuo-motor strategies used for obstacle circumvention in a virtual environment. Human Movement Science, 40:359-371, 2015

[13] M. Dicks, C. Button, and K. Davids. Examination of gaze behaviors under in situ and video simulation task constraints reveals differences in information pickup for perception and action. Attention, Perception \& Psychophysics, 72(3):706-720, 2010.

[14] P. W. Fink, P. S. Foo, and W. H. Warren. Obstacle avoidance during walking in real and virtual environments. ACM Transactions on Applied Perception (TAP), 4(1):2, 2007.

[15] T. Foulsham, E. Walker, and A. Kingstone. The where, what and when of gaze allocation in the lab and the natural environment. Vision research, 51(17):1920-1931, 2011.

[16] K. Friston, J. Ashburner, S. Kiebel, T. Nichols, and W. Penny. Statistical Parametric Mapping. Academic Press, 2007.

[17] J. Gandrud and V. Interrante. Predicting destination using head orientation and gaze direction during locomotion in vr. In Proceedings of the ACM Symposium on Applied Perception, pp. 31-38. ACM, 2016.

[18] M. Gérin-Lajoie, C. L. Richards, J. Fung, and B. J. McFadyen. Characteristics of personal space during obstacle circumvention in physical and virtual environments. Gait \& posture, 27(2):239-247, 2008.

[19] R. S. Hessels, D. C. Niehorster, M. Nyström, R. Andersson, and I. T. Hooge. Is the eye-movement field confused about fixations and saccades? a survey among 124 researchers. Royal Society open science, 5(8):180502, 2018

[20] M. Huber, Y.-H. Su, M. Krüger, K. Faschian, S. Glasauer, and J. Hermsdörfer. Adjustments of Speed and Path when Avoiding Collisions with Another Pedestrian. PLoS ONE, 9(2):e89589, 2014.

[21] J. Jovancevic, B. Sullivan, and M. Hayhoe. Control of attention and gaze in complex environments. Journal of Vision, 6(12):9-9, 2006.
[22] J. Jovancevic-Misic and M. Hayhoe. Adaptive gaze control in natural environments. Journal of Neuroscience, 29(19):6234-6238, 2009.

[23] K. Kitazawa and T. Fujiyama. Pedestrian vision and collision avoidance behavior: Investigation of the information process space of pedestrians using an eye tracker. In Pedestrian and evacuation dynamics 2008, pp. 95-108. Springer, 2010.

[24] A. Knorr, L. Willacker, J. Hermsdörfer, S. Glasauer, and M. Krüger. Influence of Person- and Situation-Specific Characteristics on Collision Avoidance Behavior in Human Locomotion. J. of Exp. Psy.: Human Perception and Performance, 42(9):1332-1343, 2016.

[25] S. Lynch, J. Pettré, J. Bruneau, R. Kulpa, A. Cretual, and A.-H. Olivier. Effect of virtual human gaze behaviour during an orthogonal collision avoidance walking task. In IEEE Virtual Reality, 2018.

[26] S. D. Lynch, R. Kulpa, L. A. Meerhoff, J. Pettre, A. Cretual, and A. H. Olivier. Collision avoidance behavior between walkers: global and local motion cues. IEEE Transactions on Visualization and Computer Graphics, PP(99):1-1, 2017. doi: 10.1109/TVCG.2017.2718514

[27] D. S. Marigold and A. E. Patla. Gaze fixation patterns for negotiating complex ground terrain. Neuroscience, 144(1):302-313, 2007.

[28] L. Meerhoff, J. Bruneau, A. Vu, A.-H. Olivier, and J. Pettré. Guided by gaze: Prioritization strategy when navigating through a virtual crowd can be assessed through gaze activity. Acta psychologica, 190:248-257, 2018.

[29] M. Moussaïd, M. Kapadia, T. Thrash, R. W. Sumner, M. Gross, D. Helbing, and C. Hölscher. Crowd behaviour during high-stress evacuations in an immersive virtual environment. Journal of The Royal Society Interface, 13(122):20160414, 2016

[30] L. Nummenmaa, J. Hyönä, and J. K. Hietanen. I'll walk this way: Eyes reveal the direction of locomotion and make passersby look and go the other way. Psychological Science, 20(12):1454-1458, 2009.

[31] A. H. Olivier, J. Bruneau, R. Kulpa, and J. Pettré. Walking with virtual people: Evaluation of locomotion interfaces in dynamic environments. IEEE Transactions on Visualization and Computer Graphics, PP(99):11, 2017. doi: 10.1109/TVCG.2017.2714665

[32] A.-H. Olivier, A. Marin, A. Crétual, A. Berthoz, and J. Pettré. Collision avoidance between two walkers: Role-dependent strategies. Gait \& Posture, 38(4):751-756, 2013.

[33] A.-H. Olivier, A. Marin, A. Crétual, and J. Pettré. Minimal predicted distance: A common metric for collision avoidance during pairwise interactions between walkers. Gait and Posture, 36(3):399-404, 2012.

[34] R. Parasuraman and M. Rizzo. Neuroergonomics: The brain at work. Oxford University Press, 2008.

[35] A. E. Patla. Understanding the roles of vision in the control of human locomotion. Gait \& Posture, 5(1):54-69, 1997.

[36] K. Pfeil, E. M. Taranta II, A. Kulshreshth, P. Wisniewski, and J. J. LaViola Jr. A comparison of eye-head coordination between virtual and physical realities. In Proceedings of the 15th ACM Symposium on Applied Perception, p. 18. ACM, 2018.

[37] M. Rubo and M. Gamer. Virtual reality as a proxy for real-life social attention? In Proceedings of the 2018 ACM Symposium on Eye Tracking Research \& Applications, p. 81. ACM, 2018.

[38] M. A. Sheik-Nainar and D. B. Kaber. The utility of a virtual reality locomotion interface for studying gait behavior. Human factors, 49(4):696-709, 2007.

[39] Q. Sun, A. Patney, L.-Y. Wei, O. Shapira, J. Lu, P. Asente, S. Zhu, M. McGuire, D. Luebke, and A. Kaufman. Towards virtual reality infinite walking: dynamic saccadic redirection. ACM Transactions on Graphics (TOG), 37(4):67, 2018.

[40] M. J. Tarr and W. H. Warren. Virtual reality in behavioral neuroscience and beyond. nature neuroscience, 5:1089, 2002.

[41] K. Varma, S. J. Guy, and V. Interrante. Assessing the relevance of eye gaze patterns during collision avoidance in virtual reality. 2017

[42] W. Warren. Visually controlled locomotion: 40 years later. Ecological Psychology, 10(3-4):177-219, 1998.

[43] W. H. Warren Jr, B. A. Kay, W. D. Zosh, A. P. Duchon, and S. Sahuc. Optic flow is used to control human walking. Nature neuroscience, 4(2):213, 2001. 